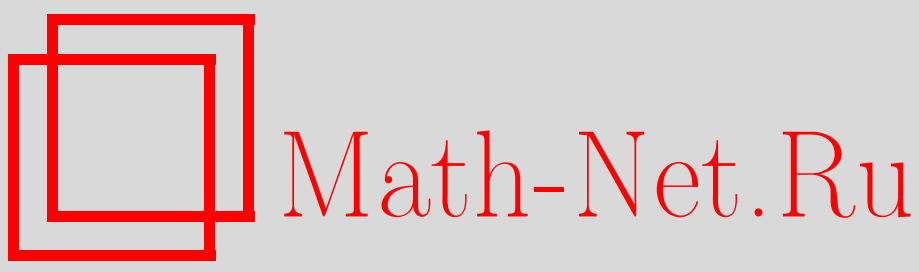

В. Ф. Кириченко, Л. В. Липагина, Киллинговы $f$-многообразия постоянного типа, Изв. РАН. Сер. матем., 1999, том 63, выпуск 5, 127-146

DOI: https://doi.org/10.4213/im261

Использование Общероссийского математического портала Math-Net.Ru подразумевает, что вы прочитали и согласны с пользовательским соглашением

http://www . mathnet.ru/rus/agreement

Параметры загрузки:

IP : 54.197 .217 .227

26 апреля 2023 г., 13:41:06 
УДК 514.76

\author{
В. Ф. Кириченко, Л. В. Липагина
}

\title{
Киллинговы $f$-многообразия постоянного типа
}

\begin{abstract}
Понятие постоянства типа было введено А. Греем в связи с изучением особенностей геометрии шестимерных приближенно келеровых многообразий и изучалось различными авторами. Это понятие естественно обобщается на случай метрических $f$-многообразий с киллинговой фундаментальной формой (киллинговых $f$-многообразий). В настоящей работе свойство постоянства типа изучается в естественно возникающем классе так называемых коммутативно-киллинговых $f$-многообразий. Изучен ряд замечательных свойств таких многообразий. Получено исчерпывающее описание коммутативно-киллинговых $f$-многообразий постоянного типа. В частности, доказано, что постоянство типа собственно коммутативно-киллинговых $f$-многообразий равносильно их локальной эквивалентности пятимерной сфере $S^{5}$, снабженной канонической слабо косимплектической структурой, индуцируемой при специальном вложении $S^{5}$ в алгебру октав Гревса-Кэли.

Библиография: 13 наименований.
\end{abstract}

Понятие постоянства типа является одним из наиболее интересных понятий геометрии приближенно келеровых многообразий. Оно было введено в рассмотрение А. Греем в [1] в связи с изучением особенностей геометрии шестимерных приближенно келеровых многообразий и изучалось различными авторами [2], [3]. Впоследствии первым из авторов настоящей работы было выяснено, что свойство постоянства типа обуславливается свойством так называемой присоединенной $K$-алгебры, близким к свойству нормированности [6]. Более того, оказалось, что свойство постоянства типа (собственного) приближенно келерова многообразия равносильно его шестимерности [6], [7].

Напомним, что почти эрмитово многообразие называется приближенно келеровым, если его фундаментальная форма является формой Киллинга. Это свойство естественно обобщается на класс метрических $f$-многообразий. Метрическая $f$-структура с киллинговой фундаментальной формой называется киллинговой $f$-структурой [4]. Известно, что $f$-многообразия киллингова типа локально (а в случае односвязности и глобально) эквивалентны произведению приближенно келерова многообразия, так называемого собственно киллингова $f$-многообразия, и риманова многообразия без дополнительной структуры [4]. Естественно, что самостоятельный интерес представляют именно собственно киллинговы $f$-многообразия. Легко видеть, что присоединенная $K$-алгебра такого многообразия абелева, т.е. ее операция композиции тривиальна. Тем не менее на таком многообразии внутренним образом определена связность, тензор аффинной деформации которой определяет на пространстве присоединенной $K$-алгебры дополнительную алгебраическую структуру, которая также может иметь постоянный тип.

(C) В.Ф. КиРИченко, Л.В. ЛипАгИнА, 1999 
В настоящей работе свойство постоянства типа изучается в естественно возникающем классе так называемых коммутативно-киллинговых $f$-многообразий. Изучен ряд замечательных свойств таких многообразий. Получена исчерпьвающая характеристика коммутативно-киллинговых $f$-многообразий постоянного типа. В частности, нами доказано, что постоянство типа собственно коммутативно-киллинговых $f$-многообразий равносильно их локальной эквивалентности пятимерной сфере $S^{5}$, снабженной канонической слабо косимплектической структурой, индуцируемой при специальном вложении $S^{5}$ в алгебру $\mathbf{O}$ октав Гревса-Кэли [5].

Пусть $M$ - гладкое многообразие, $\mathrm{X}(M)-C^{\infty}(M)$-модуль гладких векторных полей на $M, d$ - оператор внешнего дифференцирования. Все многообразия, тензорные поля и т.п. объекты предполагаются гладкими класса $C^{\infty}$. Напомним [9], что метрической $f$-структурой на $M$ называется пара $\mathscr{S}=(f, g)$ тензорных полей на $M$, где $f$ - тензор типа $(1,1)$, называемый структурным әндоморфизмом, $f^{3}+f=0, g=\langle\cdot, \cdot\rangle$ - риманова метрика. При этом $\langle f X, Y\rangle+\langle X, f Y\rangle=0$, $X, Y \in \mathrm{X}(M)$. Многообразие, на котором фиксирована такая структура, называется метрическим $f$-многообразием. Число $\operatorname{dim} \operatorname{ker} f$ называется дефектом структуры и обозначается $\operatorname{def} f$ или $\operatorname{def} M$. Непосредственно проверяется, что тензор $\Omega(X, Y)=\langle X, f Y\rangle$ кососимметричен, т.е. является 2-формой на $M$. Он называется фундаментальной формой структуры. Если $\Omega$ - форма Киллинга, т.е. $\nabla \Omega=d \Omega$, где $\nabla$ - риманова связность на $M, \mathscr{S}$ назьвается киллинговой $f$-структурой [4]. Важными примерами киллинговых $f$-структур являются $n p u$ ближенно келеровы структуры ( $f$ - почти комплексная структура, $\operatorname{def} f=0)$, а также слабо косимплектические структуры ( $f$-почти контактная структура, $\operatorname{def} f=1)$.

Пусть $M$ - метрическое $f$-многообразие. Тогда $\mathrm{X}(M)=\mathscr{L} \oplus \mathscr{M}$, где $\mathscr{L}=\operatorname{Im} f$, $\mathscr{M}=\operatorname{ker} f$-взаимно ортогональные распределения, которые называются первы.м и вторым фундаментальными распределениями структуры соответственно. Очевидно, эндоморфизмы $\mathrm{I}=-f^{2}$ и $\mathrm{m}=\mathrm{id}+f^{2}$ являются взаимно дополнительными проекторами на распределения $\mathscr{L}$ и $\mathscr{M}$ соответственно. Заметим, что сужение $(F, g) f$-структуры $\mathscr{S}$ на $\mathscr{L}$ является почти эрмитовой структурой, т.е. $F^{2}=-\mathrm{id},\langle F X, F Y\rangle=\langle X, Y\rangle, X, Y \in \mathscr{L}$. Это позволяет к $\mathscr{S}$ внутренним образом присоединить $G$-структуру со структурной группой $U(n) \times O(m, \mathbb{R})$, где $n-$ комплексная размерность распределения $\mathscr{L}, m$ - размерность распределения $\mathscr{M}$.

Именно, пусть $p \in M$. Построим ортонормированньй базис $\left(e_{1}, \ldots, e_{n}\right.$, $\left.f e_{1}, \ldots, f e_{n}\right)$ пространства $\mathscr{L}_{p}$ и ортонормированньй базис $\left(e_{2 n+1}, \ldots, e_{2 n+m}\right)$ пространства $\mathscr{M}_{p}$. Тогда $\left(p, e_{1}, \ldots, e_{n}, f e_{1}, \ldots, f e_{n}, e_{2 n+1}, \ldots, e_{2 n+m}\right)$ - ортонормированный репер касательного пространства $T_{p}(M)$. Такой репер будем называть вещественно адаптированным репером. С другой стороны, пусть $\mathscr{L}^{c}=\mathscr{L} \otimes \mathbb{C}$ - комплексификация распределения $\mathscr{L}$. В ней внутренним образом определены два взаимно дополнительных проектора $\sigma=(1 / 2)(\mathrm{id}-\sqrt{-1} f)$ и $\bar{\sigma}=$ $(1 / 2)(\mathrm{id}+\sqrt{-1} f)$ на собственные подмодули эндоморфизма $f$, отвечающие собственным значениям $\sqrt{-1}$ и $-\sqrt{-1}$ соответственно. Следовательно, можно построить репер $\left(p, \varepsilon_{1}, \ldots, \varepsilon_{n}, \varepsilon_{\hat{1}}, \ldots, \varepsilon_{\hat{n}}, \varepsilon_{2 n+1}, \ldots, \varepsilon_{2 n+m}\right)$ комплексификации пространства $T_{p}(M)$, где $\varepsilon_{a}=\sqrt{2} \sigma\left(e_{a}\right), \varepsilon_{\hat{a}} \equiv \varepsilon^{a}=\sqrt{2} \bar{\sigma}\left(e_{a}\right), \varepsilon_{\alpha}=e_{\alpha}$, 
состоящий из собственных векторов оператора $f_{p}$. Такой репер называется $A$-репером. Легко видеть, что матрицы компонент тензоров $f_{p}$ и $g_{p}$ в $A$-репере имеют соответственно вид

$$
\left(f_{j}^{i}\right)=\left(\begin{array}{ccc}
\sqrt{-1} I_{n} & 0 & 0 \\
0 & -\sqrt{-1} I_{n} & 0 \\
0 & 0 & Z_{m}
\end{array}\right), \quad\left(g_{i j}\right)=\left(\begin{array}{ccc}
0 & I_{n} & 0 \\
I_{n} & 0 & 0 \\
0 & 0 & Z_{m}
\end{array}\right)
$$

где $I_{n}$ и $Z_{m}$ - единичная и нулевая матрицы порядка $n$ и $m$ соответственно. Хорошо известно [11], что совокупность таких реперов определяет $G$-структуру на $M$ со структурной группой $U(n) \times O(m, \mathbb{R})$, представленной матрицами вида $\left(\begin{array}{ccc}A & 0 & 0 \\ 0 & \bar{A} & 0 \\ 0 & 0 & B\end{array}\right)$, где $A \in U(n), \quad B \in O(m, \mathbb{R})$. Эта $G$-структура называется присоединенной. На протяжении всей работы будем подразумевать, что индексы $i, j, k, \ldots$ пробегают значения от 1 до $2 n+m$, индексы $a, b, c, d, f, g, \ldots$ - значения от 1 до $n$ и индексы $\alpha, \beta, \gamma, \ldots$ - значения от $2 n+1$ до $2 n+m$. Положим $\hat{a}=a+n, \widehat{\alpha}=\alpha$. Поскольку $f$ и $g$ - тензоры типов $(1,1)$ и $(2,0)$ соответственно, их компоненты на пространстве расслоения всех комплексных реперов над $M$ удовлетворяют уравнениям:

$$
\begin{aligned}
& \text { 1) } d f_{j}^{i}+f_{k}^{i} \omega_{j}^{k}-f_{j}^{k} \omega_{k}^{i}=f_{j, k}^{i} \omega^{k} ; \\
& \text { 2) } d g_{i j}+g_{k j} \omega_{i}^{k}+g_{i k} \omega_{j}^{k}=g_{i j, k} \omega^{k} ;
\end{aligned}
$$

где $\left\{\omega^{i}\right\},\left\{\omega_{j}^{i}\right\}$ - компоненты форм смешения и римановой связности $\nabla$ соответственно, $f_{j, k}^{i}, g_{i j, k}$ - компоненты ковариантного дифференциала тензоров $f$ и $g$ в этой связности соответственно. Более того, в силу определения римановой связности $\nabla g=0$, и, значит,

$$
g_{i j, k}=0 .
$$

C учетом (1) и (3) соотношения (2) на пространстве присоединенной $G$-структуры перепишутся в форме:
1) $f_{b, k}^{a}=0 ;$ 2) $f_{\hat{b}, k}^{\hat{a}}=0$;
3) $f_{\beta, k}^{\alpha}=0$;
4) $\omega_{\hat{b}}^{a}=-\frac{1}{2} \sqrt{-1} f_{\hat{b}, k}^{a} \omega^{k}$
5) $\omega_{b}^{\hat{a}}=\frac{1}{2} \sqrt{-1} f_{b, k}^{\hat{a}} \omega^{k}$;
6) $\omega_{\alpha}^{a}=-\sqrt{-1} f_{\alpha, k}^{a} \omega^{k}$;
7) $\omega_{\alpha}^{\hat{a}}=\sqrt{-1} f_{\alpha, k}^{\hat{a}} \omega^{k}$
8) $\omega_{a}^{\alpha}=\sqrt{-1} f_{a, k}^{\alpha} \omega^{k}$;
9) $\omega_{\hat{a}}^{\alpha}=-\sqrt{-1} f_{\hat{a}, k}^{\alpha} \omega^{k}$;
10) $\omega_{j}^{i}+\omega_{\hat{\imath}}^{\hat{\jmath}} \equiv 0(\bmod n)$.

Кроме того, заметим, что в силу вешественности соответствующих форм и тензоров имеем

$$
\bar{\omega}^{i} \equiv \omega^{\hat{\imath}} \quad(\bmod n), \quad \bar{\omega}_{j}^{i} \equiv \omega_{\hat{\jmath}}^{\hat{\imath}} \quad(\bmod n), \quad \overline{\nabla f}_{j, k}^{i} \equiv \nabla f_{\hat{\jmath}, \hat{k}}^{\hat{\imath}} \quad(\bmod n),
$$


где $t \rightarrow \bar{t}$ - оператор комплексного сопряжения. Пусть, в частности, $M-$ киллингово $f$-многообразие. Очевидно, условие киллинговости формы $\Omega$ равносильно соотношениям

$$
f_{j, k}^{i}+f_{k, j}^{i}=0
$$

С учетом (4) и (5) получаем:

$$
\begin{array}{ll}
\text { 1) } \omega_{\hat{b}}^{a}=-B^{a b c} \omega_{c}-\frac{1}{2} B_{\alpha}^{a b} \omega^{\alpha} ; & \text { 2) } \omega_{b}^{\hat{a}}=-B_{a b c} \omega^{c}-\frac{1}{2} B_{\alpha a b} \omega^{\alpha} ; \\
\text { 3) } \omega_{\alpha}^{a}=B_{\alpha}^{a b} \omega_{b} ; & \text { 4) } \omega_{a}^{\alpha}=-B_{a b}^{\alpha} \omega^{b} \\
\text { 5) } \omega_{\alpha}^{\hat{a}}=B_{\alpha a b} \omega^{b} ; & \text { 6) } \omega_{\hat{a}}^{\alpha}=-B^{\alpha a b} \omega_{b} \\
\text { 7) } \omega_{b}^{a}+\omega_{\hat{a}}^{\hat{b}}=0 ; & \text { 8) } \omega_{\beta}^{\alpha}+\omega_{\alpha}^{\beta}=0 ;
\end{array}
$$

где $B^{a b c}=(\sqrt{-1} / 2) f_{\hat{b}, \hat{c}}^{a}, B_{a b c}=-(\sqrt{-1} / 2) f_{b, c}^{\hat{a}}-$ системы функций на пространстве присоединенной $G$-структуры, кососимметричные по всем индексам; $B_{\alpha}^{a b} \equiv$ $B^{\alpha a b}=\sqrt{-1} f_{\hat{b}, \alpha}^{a}, B_{\alpha a b} \equiv B_{a b}^{\alpha}=-\sqrt{-1} f_{b, \alpha}^{\hat{a}}-$ системы функций на пространстве присоединенной $G$-структуры, кососимметричные по первой паре индексов; $\omega_{i}=g_{i j} \omega^{j}$.

С учетом (6) нетрудно подсчитать, что первая группа структурных уравнений римановой связности

$$
d \omega^{i}=\omega_{j}^{i} \wedge \omega^{j}
$$

на пространстве присоединенной $G$-структуры киллингова $f$-многообразия запишется в следующей форме, называемой первой группой структурных уравнений киллингова $f$-многообразия:

$$
\begin{aligned}
d \omega^{a} & =\omega_{b}^{a} \wedge \omega^{b}+B^{a b c} \omega_{b} \wedge \omega_{c}+\frac{3}{2} B_{\alpha}^{a b} \omega_{b} \wedge \omega^{\alpha}, \\
d \omega_{a} & =-\omega_{a}^{b} \wedge \omega_{b}+B_{a b c} \omega^{b} \wedge \omega^{c}+\frac{3}{2} B_{a b}^{\alpha} \omega^{b} \wedge \omega_{\alpha}, \\
d \omega^{\alpha} & =\omega_{\beta}^{\alpha} \wedge \omega^{\beta}+B_{a b}^{\alpha} \omega^{a} \wedge \omega^{b}+B^{\alpha a b} \omega_{a} \wedge \omega_{b} .
\end{aligned}
$$

Применяя оператор комплексного сопряжения к обеим частям уравнений (7) и сравнивая с соответствующими уравнениями (7), с учетом линейной независимости базисных форм получим:
1) $\omega_{b}^{a}+\bar{\omega}_{a}^{b}=0$
2) $\bar{\omega}_{\beta}^{\alpha}=\omega_{\beta}^{\alpha}$
3) $\bar{B}_{a b c}=B^{a b c}$
4) $\bar{B}_{a b}^{\alpha}=B_{\alpha}^{a b}$.

Стандартная процедура дифференциального продолжения соотношений (7) приводит ко второй группе структурных уравнений киллинговой $f$-структурь:

1) $d \omega_{b}^{a}=\omega_{c}^{a} \wedge \omega_{b}^{c}+\left(2 B^{a d h} B_{h b c}+\frac{3}{2} B_{\alpha}^{a d} B_{b c}^{\alpha}+A_{b c}^{a d}\right) \omega^{c} \wedge \omega_{d}$

$$
+\frac{9}{4} B_{[\alpha}^{a h} B_{\beta] h b} \omega^{\alpha} \wedge \omega^{\beta}
$$

2) $d \omega_{\beta}^{\alpha}=\omega_{\gamma}^{\alpha} \wedge \omega_{\beta}^{\gamma}+6 B_{[\alpha}^{d h} B_{\beta] c h} \omega^{c} \wedge \omega_{d}+A_{\beta \gamma \delta}^{\alpha} \omega^{\gamma} \wedge \omega^{\delta}$, 
где $\left\{A_{b c}^{a d}\right\}$ - семейство функций на пространстве присоединенной $G$-структуры, служащих компонентами так называемого тензора кривизны присоединенной $Q$-алгебры [10], [11], или структурного тензора третьего рода, причем:

1) $A_{[b c]}^{a d}=0 ; \quad$ 2) $\left.A_{b c}^{[a d]}=0 ; \quad 3\right) B^{a d h} B_{h c}^{\alpha}=0$;

$\left\{A_{\beta \gamma \delta}^{\alpha}\right\}$ - семейство функций на пространстве присоединенной $G$-структуры, служащих компонентами так называемого структурного тензора четвертого рода, обладающее классическими свойствами симметрии тензора Римана-Кристоффеля. Кроме того:

1) $d B^{a b c}-B^{h b c} \omega_{h}^{a}-B^{a h c} \omega_{h}^{b}-B^{a b h} \omega_{h}^{c}=B^{a b c h} \omega_{h}$;

2) $d B_{a b c}+B_{h b c} \omega_{a}^{h}+B_{a h c} \omega_{b}^{h}+B_{a b h} \omega_{c}^{h}=B_{a b c h} \omega^{h}$;

3) $d B^{\alpha a b}-B^{\beta a b} \omega_{\beta}^{\alpha}-B^{\alpha h b} \omega_{h}^{a}-B^{\alpha a h} \omega_{h}^{b}=0$;

4) $d B_{\alpha a b}+B_{\beta a b} \omega_{\alpha}^{\beta}+B_{\alpha h b} \omega_{a}^{h}+B_{\alpha a h} \omega_{b}^{h}=0$;

где $B^{a b c d}, B_{a b c d}$ - подходяшие функции на пространстве присоединенной $G$-структуры. Отсюда, в частности, следует, что наборы функций $\left\{B^{a b c}\right\}$ и $\left\{B_{a b c}\right\},\left\{B^{\alpha a b}\right\}$ и $\left\{B_{\alpha a b}\right\}$ являются наборами компонент вещественных тензоров на $M$, которыемы назовем структурными тензорами первого и второго рода соответственно. Здесь и в дальнейшем по индексам, заключенным в квадратные (соответственно круглые) скобки, подразумевается альтернирование (соответственно симметризация).

Сравнивая соотношения (9) со второй группой структурных уравнений римановой связности

$$
d \omega_{j}^{i}=\omega_{k}^{i} \wedge \omega_{j}^{k}+\frac{1}{2} R_{j k l}^{i} \omega^{k} \wedge \omega^{l},
$$

где $R_{j k l}^{i}$ - компоненты тензора Римана-Кристоффеля, в силу линейной независимости базисных форм получим, что на пространстве присоединенной $G$-структуры:

$$
\begin{array}{ll}
\text { 1) } R_{\hat{a} b c \hat{d}}=B^{a d h} B_{h b c}+\frac{1}{2} B^{\alpha a d} B_{\alpha b c}+A_{b c}^{a d} ; \\
\text { 2) } R_{\hat{a} b \alpha \beta}=4 B_{[\alpha}^{a h} B_{\beta] h b} ; & \text { 3) } R_{\beta \gamma \delta}^{\alpha}=2 A_{\beta \gamma \delta}^{\alpha} .
\end{array}
$$

Кроме того, дифференциальные продолжения соотношений (6) дают:

$$
\begin{aligned}
& \text { 1) } R_{a b c d}=-B_{a c d b} ; \quad \text { 2) } R_{\hat{a} \hat{b} c d}=-2 B^{a b h} B_{h c d}-B^{\alpha a b} B_{\alpha c d} ; \\
& \text { 3) } R_{\hat{a} \alpha b \beta}=\frac{3}{2} B_{\alpha}^{a h} B_{\beta h b}-\frac{1}{2} B_{\beta}^{a h} B_{\alpha h b}
\end{aligned}
$$

плюс соотношения, полученные из (12) и (13) с учетом классических свойств симметрии и вещественности тензора Римана-Кристоффеля. Остальные компоненты тензора $R$ равны нулю.

С учетом (12) и (13) получаем выражение компонент тензора $r$ Риччи на пространстве присоединенной $G$-структуры:

$$
\text { 1) } r_{\hat{a} b}=3 B^{a d h} B_{b d h}+\frac{5}{2} B^{\alpha a h} B_{\alpha b h}-A_{b h}^{a h}
$$


2) $r_{\alpha \beta}=2\left(B_{(\alpha}^{d h} B_{\beta) d h}+A_{\alpha \beta \gamma}^{\gamma}\right)$;

плюс соотношения, полученные с учетом симметричности тензора Риччи. Остальные компоненты этого тензора равны нулю.

На киллинговом $f$-многообразии $M$ внутренним образом определены две связности. Прежде всего из соотношений (9) и (8) в силу теоремы Картана-Лаптева следует, что форма

$$
\zeta=\left(\zeta_{j}^{i}\right)=\left(\begin{array}{ccc}
\omega_{b}^{a} & 0 & 0 \\
0 & \omega_{\hat{b}}^{\hat{a}} & 0 \\
0 & 0 & \omega_{\beta}^{\alpha}
\end{array}\right)
$$

на пространстве присоединенной $G$-структуры является формой некоторой связности $\widehat{\nabla}$ на $M$, которую мы назовем канонической связностью. Тензор $S(X, Y)=$ $\widehat{\nabla}_{X} Y-\nabla_{X} Y$ аффинной деформации этой связности определяет в модуле $\mathrm{X}(M)$ структуру алгебры с операцией композиции $X \bullet Y=S(X, Y), X, Y \in \mathrm{X}(M)$. Из формул (2) следует, что в этой связности $\widehat{\nabla} g=0, \widehat{\nabla} f=0$; в частности, справедливо тождество

$$
\langle X \bullet Y, Z\rangle+\langle Y, X \bullet Z\rangle=0, \quad X, Y, Z \in \mathrm{X}(M) .
$$

Далее, напомним [9], что в модуле $\mathrm{X}(M)$ метрического $f$-многообразия $M$ внутренним образом определена структура $Q$-алгебры с операцией композиции $X * Y=T(X, Y)$, где $T(X, Y)=\frac{1}{4}\left\{f \nabla_{f X}(f) f Y-f \nabla_{f^{2} X}(f) f^{2} Y\right\}-$ композиционный тензор $Q$-алгебры. В случае когда $M$ - киллингово $f$-многообразие, эта $Q$-алгебра, очевидно, является $K$-алгеброй, т.е. она антикоммутативна. Более того, ковариантно продифференцируем соотношение $f \circ f \circ f=-f$ :

$$
\nabla_{X}(f)\left(f^{2} Y\right)+f \nabla_{X}(f)(f Y)+f^{2} \nabla_{X}(f) Y=-\nabla_{X}(f) Y .
$$

Применив к обеим частям этого тождества эндоморфизм $f$, получим

$$
f \nabla_{X}(f)\left(f^{2} Y\right)+f^{2} \nabla_{X}(f)(f Y)=0 .
$$

С учетом этого легко проверить, что композиционный тензор $K$-алгебры, присоединенной к киллингову $f$-многообразию, вычисляется по формуле

$$
\mathscr{T}(X, Y)=\frac{1}{2} f \nabla_{f X}(f)(f Y) .
$$

Рассматривая его как тензор аффинной деформации, получаем еще одну связность $\widetilde{\nabla}_{X} Y=\nabla_{X} Y+\mathscr{T}(X, Y)$ на $M$, назьваемую присоединенной [9].

Пусть $\mathscr{T}$ - тензор аффинной деформации от присоединенной связности к связности канонической. Он также определяет в модуле $\mathrm{X}(M)$ структуру алгебры с операцией композиции $X \diamond Y=T(X, Y)$. Очевидно,

$$
X \bullet Y=X * Y+X \diamond Y, \quad X, Y \in \mathrm{X}(M) .
$$

В частности, в силу (16) и определения $Q$-алгебры [9] справедливо тождество

$$
\langle X \diamond Y, Z\rangle+\langle Y, X \diamond Z\rangle=0, \quad X, Y, Z \in \mathrm{X}(M) .
$$


ЗАмечание 1 . Соотношения (16) и (18) показывают, что модуль $\mathrm{X}(M)$ относительно операций $\bullet$ и $\diamond$ является вещественной $Q$-алгеброй. Назовем ее канонической $Q$-алгеброй многообразия $M$ и обозначим $\mathscr{W}$.

С учетом (6) и тождеств $d Y^{i}-Y^{j} \omega_{j}^{i}=\nabla_{j} Y^{i} \omega^{j}, d Y^{i}-Y^{j} \theta_{j}^{i}=\widehat{\nabla}_{j} Y^{i} \omega^{j}$ несложно проверить, что на пространстве присоединенной $G$-структуры тензор $S$ имеет компоненты

$$
S_{\hat{b} \hat{c}}^{a}=B^{a b c}, \quad S_{\hat{b} \alpha}^{a}=B_{\alpha}^{a b}, \quad S_{\alpha \hat{b}}^{a}=-\frac{1}{2} B_{\alpha}^{a b}, \quad S_{\hat{b} \hat{c}}^{\alpha}=B^{\alpha b c}
$$

и формулы комплексно сопряженные. Остальные компоненты этого тензора равны нулю. С другой стороны, с учетом (17) находим, что на пространстве присоединенной $G$-структуры тензор $\mathscr{T}$ имеет компоненты $\mathscr{T}_{\hat{b} \hat{c}}^{a}=B^{a b c}$ и формулы комплексно сопряженные. Остальные компоненты этого тензора равны нулю. Следовательно, на пространстве присоединенной $G$-структуры тензор $T$ имеет компоненты

$$
T_{\hat{b} \alpha}^{a}=B_{\alpha}^{a b}, \quad T_{\alpha \hat{b}}^{a}=-\frac{1}{2} B_{\alpha}^{a b}, \quad T_{\hat{b} \hat{c}}^{\alpha}=B^{\alpha b c}
$$

и формулы комплексно сопряженные. Остальные компоненты этого тензора равны нулю. Эти соотношения (а также $\left.\left(10_{3}\right)\right)$ показывают, в частности, что справедливо

ПРЕДЛОЖЕНИЕ 1. В сделанных обозначениях операция $\diamond$ обладает свойствами:

1) $\mathrm{I}(X \diamond Y)=\mathrm{I}(X) \diamond \mathrm{m}(Y)+\mathrm{m}(X) \diamond \mathrm{I}(Y)$;

2) $\mathrm{I}(X) \diamond \mathrm{m}(Y)=-2 \mathrm{~m}(Y) \diamond \mathrm{I}(X)$

3) $\mathrm{I}(X) \diamond \mathrm{I}(Y)=-\mathrm{I}(Y) \diamond \mathrm{I}(X)=\mathrm{m}(X \diamond Y)$;

4) $\mathrm{m}(X) \diamond \mathrm{m}(Y)=0$

5) $\mathrm{I}(f X) \diamond \mathrm{m}(Y)=-f(\mathrm{I}(X) \diamond \mathrm{m}(Y))$;

6) $\mathrm{I}(f X) \diamond \mathrm{I}(f Y)=-\mathrm{I}(X) \diamond \mathrm{I}(Y)$;

7) $X *(Y \diamond Z)=X \diamond(Y * Z)=(Y * Z) \diamond X=0$; әде $X, Y, Z \in \mathrm{X}(M)$.

В частности, $\mathscr{L} \diamond \mathscr{L} \subset \mathscr{M}, \mathscr{L} \diamond \mathscr{M}=\mathscr{M} \diamond \mathscr{L} \subset \mathscr{L}, \mathscr{M} \diamond \mathscr{M}=\{0\}$.

Определим в $\mathscr{W}$ эндоморфизмы $L_{X}: \mathscr{W} \rightarrow \mathscr{W}$ левого сдвига на элемент $X \in \mathscr{W}$ по формуле $L_{X}(Y)=X \diamond Y$. Пусть $u, v, w \in \mathscr{L}, a, b, c \in \mathscr{M}$. В силу предложения 1 определено сужение на $\mathscr{M}$ эндоморфизма $L_{u} \circ L_{v}$. Это позволяет определить на $\mathscr{L}$ симметричную билинейную форму $\Theta(u, v)=-\operatorname{tr}\left(L_{u} \circ L_{v}\right)$, а также самосопряженный эндоморфизм $\theta \in$ End $\mathscr{L}$, определенный тождеством $\Theta(u, v)=\langle u, \theta(v)\rangle$.

ТЕорема 1. Эндоморфизм $\theta$ обладает следующими свойствами:

1) в произвольном A-репере

$$
\theta_{p}(u)=-\sum_{\alpha}\left(u \diamond \varepsilon_{\alpha}\right) \diamond \varepsilon_{\alpha}, \quad p \in M, \quad u \in \mathscr{L}
$$

2) $\Theta$ - положительно полуопределенная форма; 
3) в произвольном А-репере эндоморфизм $\theta_{p}$ задается матрицей

$$
\left(\begin{array}{cc}
\theta_{c}^{b} & 0 \\
0 & \theta_{\hat{c}}^{b}
\end{array}\right)
$$

əде $\theta_{c}^{b}=B^{\alpha h b} B_{\alpha h c}, \theta_{\hat{c}}^{\hat{b}}=\bar{\theta}_{c}^{b}=\theta_{b}^{c}$

4) $\theta \circ f=f \circ \theta$;

5) $\widehat{\nabla} \theta=0$;

6) $\mathscr{L}=\operatorname{Im} \theta \oplus \operatorname{ker} \theta$, причем $\operatorname{Im} \theta \perp \operatorname{ker} \theta$;

7) $\operatorname{ker} \theta \diamond \mathscr{M}=\mathscr{M} \diamond \operatorname{ker} \theta=\{0\}$;

8) $\mathscr{L} \diamond \mathscr{M}=\mathscr{M} \diamond \mathscr{L}=\operatorname{Im} \theta$.

ДокАЗАТЕЛЬСТво. 1) Согласно (18) и п. 3) предложения 1

$$
\begin{aligned}
-\sum_{\alpha}\left\langle\left(u \diamond \varepsilon_{\alpha}\right) \diamond \varepsilon_{\alpha}, v\right\rangle & =\sum_{\alpha}\left\langle\varepsilon_{\alpha},\left(u \diamond \varepsilon_{\alpha}\right) \diamond v\right\rangle=-\sum_{\alpha}\left\langle v \diamond\left(u \diamond \varepsilon_{\alpha}\right), \varepsilon_{\alpha}\right\rangle \\
& =-\operatorname{tr}\left(L_{v} \circ L_{u}\right)=\Theta(v, u)=\langle v, \theta(u)\rangle=\langle\theta(u), v\rangle,
\end{aligned}
$$

откуда в силу невырожденности метрики следует (20) (здесь и в дальнейшем индекс $p$ будем опускать).

2) В силу (18), (20) и п. 2) предложения 1

$$
\Theta(u, u)=\langle u, \theta(u)\rangle=\sum_{\alpha}\left\|u \diamond \varepsilon_{\alpha}\right\|^{2} \geqslant 0 .
$$

3) В силу (19)

$$
\begin{aligned}
& (u \diamond a)^{b}=T_{\hat{c} \alpha}^{b} u^{\hat{c}} a^{\alpha}=B_{\alpha}^{b c} u_{c} a^{\alpha}, \\
& ((u \diamond a) \diamond b)^{a}=T_{\hat{b} \alpha}^{a}(u \diamond a)^{\hat{b}} b^{\alpha}=B_{\alpha}^{a b} B_{b c}^{\beta} u^{c} a_{\beta} b^{\alpha} .
\end{aligned}
$$

Положив здесь $a=b=\varepsilon_{\alpha}$ и суммируя по $\alpha$, с учетом (20) получим (21).

4) Немедленно следует из (1) и (21).

5) Немедленно следует из $\left(11_{3,4}\right),(15)$ и п. 3$)$.

6) Немедленно следует из самосопряженности эндоморфизма $\theta$.

7) Первое равенство немедленно следует из п. 2) предложения 1 . Пусть теперь $u \in \operatorname{ker} \theta$. Тогда в силу (22) $u \diamond \varepsilon_{\alpha}=0, \alpha=2 n+1, \ldots, 2 n+m$. Следовательно, $u \diamond a=0(a \in \mathscr{M})$, и, значит, $\operatorname{ker} \theta \diamond \mathscr{M}=\mathscr{M} \diamond \operatorname{ker} \theta=\{0\}$.

8) Первое равенство немедленно следует из п. 2) предложения 1 . Пусть теперь $u \in \mathscr{M} \diamond \mathscr{L}$. Тогда $u=\sum_{k=1}^{N} a_{k} \diamond v_{k}$, где $a_{k} \in \mathscr{M}, v_{k} \in \mathscr{L}$. Пусть $w \in \operatorname{ker} \theta$. Тогда в силу (18) и п. 7)

$$
\langle w, u\rangle=\sum_{k=1}^{N}\left\langle w, a_{k} \diamond v_{k}\right\rangle=-\sum_{k=1}^{N}\left\langle a_{k} \diamond w, v_{k}\right\rangle=0 .
$$

Следовательно, $\mathscr{M} \diamond \mathscr{L} \perp \operatorname{ker} \theta$. Но тогда в силу п. 6$) \mathscr{M} \diamond \mathscr{L} \subset \operatorname{Im} \theta$. Обратно: если $w \in \operatorname{Im} \theta$, то в силу (20) и предложения $1 w=\theta(u)=-\sum_{\alpha}\left(u \diamond \varepsilon_{\alpha}\right) \diamond \varepsilon_{\alpha} \in \mathscr{M} \diamond \mathscr{L}$. Следовательно, $\mathscr{L} \diamond \mathscr{M}=\mathscr{M} \diamond \mathscr{L}=\operatorname{Im} \theta$.

Аналогично, в силу предложения 1 определено сужение на $\mathscr{L}$ эндоморфизма $L_{a} \circ L_{b}$. Это позволяет определить на $\mathscr{M}$ симметричную билинейную форму $\Psi(a, b)=-\operatorname{tr}\left(L_{a} \circ L_{b}\right)$, а также самосопряженный эндоморфизм $\psi \in \operatorname{End} \mathscr{M}$, определенный тождеством $\Psi(a, b)=\langle a, \psi(b)\rangle$. 
ТЕОРема 2. Эндоморфизм $\psi$ обладает следующими свойствами:

1) в произвольном A-репере (соответственно вещественно-адаптированном репере)

$$
\psi(b)=-\frac{1}{2}\left(\left(b \diamond \varepsilon^{a}\right) \diamond \varepsilon_{a}+\left(b \diamond \varepsilon_{a}\right) \diamond \varepsilon^{a}\right)=-\sum_{a}\left(b \diamond e_{a}\right) \diamond e_{a} ;
$$

2) $\Psi$ - положительно полуопределенная форма;

3) в произвольном А-репере эндоморфизм $\psi_{p}$ задается матрицей

$$
\left(\psi_{\beta}^{\alpha}\right), \quad \psi_{\beta}^{\alpha}=\frac{1}{4}\left(B^{\alpha h c} B_{\beta h c}+B_{h c}^{\alpha} B_{\beta}^{h c}\right)
$$

4) $\psi \circ f=f \circ \psi=0$;

5) $\widehat{\nabla} \psi=0$;

6) $\mathscr{M}=\operatorname{Im} \psi \oplus \operatorname{ker} \psi$, причем $\operatorname{Im} \psi \perp \operatorname{ker} \psi$;

7) $\operatorname{ker} \psi \diamond \mathscr{L}=\mathscr{L} \diamond \operatorname{ker} \psi=\{0\}$;

8) $\mathscr{L} \diamond \mathscr{L}=\operatorname{Im} \psi$.

ДоКАЗАТЕЛЬСтво. 1) Согласно (18) и п. 2) предложения 1

$$
\begin{aligned}
& -\left\langle\left(b \diamond \varepsilon^{a}\right) \diamond \varepsilon_{a}, c\right\rangle-\left\langle\left(b \diamond \varepsilon_{a}\right) \diamond \varepsilon^{a}, c\right\rangle=\left\langle\varepsilon_{a},\left(b \diamond \varepsilon^{a}\right) \diamond c\right\rangle+\left\langle\varepsilon^{a},\left(b \diamond \varepsilon_{a}\right) \diamond c\right\rangle \\
& \quad=-2\left\langle c \diamond\left(b \diamond \varepsilon^{a}\right), \varepsilon_{a}\right\rangle-2\left\langle c \diamond\left(b \diamond \varepsilon_{a}\right), \varepsilon^{a}\right\rangle=-2 \operatorname{tr}\left(L_{c} \circ L_{b}\right) \\
& \quad=2 \Psi(c, b)=2\langle c, \psi(b)\rangle=2\langle\psi(b), c\rangle .
\end{aligned}
$$

Аналогично, с учетом пп. 5), 6) предложения $1\left(b \diamond f\left(e_{a}\right)\right) \diamond f\left(e_{a}\right)=-f\left(b \diamond e_{a}\right) \diamond$ $f\left(e_{a}\right)=\left(b \diamond e_{a}\right) \diamond e_{a}$ и, значит,

$$
\begin{aligned}
-2 \sum_{a}\left\langle\left(b \diamond e_{a}\right) \diamond e_{a}, c\right\rangle & =-\sum_{a}\left(\left\langle\left(b \diamond e_{a}\right) \diamond e_{a}, c\right\rangle+\left\langle\left(b \diamond f\left(e_{a}\right)\right) \diamond f\left(e_{a}\right), c\right\rangle\right) \\
& =\sum_{a}\left(\left\langle e_{a},\left(b \diamond e_{a}\right) \diamond c\right\rangle+\left\langle f\left(e_{a}\right),\left(b \diamond f\left(e_{a}\right)\right) \diamond c\right\rangle\right) \\
& =-2 \sum_{a}\left(\left\langle c \diamond\left(b \diamond e_{a}\right), e_{a}\right\rangle+\left\langle c \diamond\left(b \diamond f\left(e_{a}\right)\right), f\left(e_{a}\right)\right\rangle\right) \\
& =-2 \operatorname{tr}\left(L_{c} \circ L_{b}\right)=2 \Psi(c, b)=2\langle c, \psi(b)\rangle=2\langle\psi(b), c\rangle,
\end{aligned}
$$

откуда в силу невырожденности метрики следует (23).

2) В силу (18), (23) и пп. 2), 3) предложения 1

$$
\Psi(b, b)=\langle b, \psi(b)\rangle=2 \sum_{a}\left\|b \diamond e_{a}\right\|^{2} \geqslant 0 .
$$

3) В силу (19)

$$
\begin{aligned}
(a \diamond u)^{b} & =T_{\alpha \hat{c}}^{b} a^{\alpha} u^{\hat{c}}=-\frac{1}{2} B_{\alpha}^{b c} a^{\alpha} u_{c} \\
((a \diamond u) \diamond v)^{\alpha} & =T_{\hat{b} \hat{c}}^{\alpha}(a \diamond u)^{\hat{b}} v^{\hat{c}}+T_{b c}^{\alpha}(a \diamond u)^{b} v^{c} \\
& =-\frac{1}{2}\left(B^{\alpha b c} B_{\beta b d} a^{\beta} u^{d} v_{c}+B_{b c}^{\alpha} B_{\beta}^{b d} a^{\beta} u_{d} v^{c}\right) .
\end{aligned}
$$


Положив здесь сначала $u=\varepsilon^{a}, v=\varepsilon_{a}$, а потом $u=\varepsilon_{a}, v=\varepsilon^{a}$ и суммируя по $a$, с учетом (23) получим (24).

4) Немедленно следует из (1).

5) Немедленно следует из $\left(11_{3,4}\right),(15)$ и п. 3$)$.

6) Немедленно следует из самосопряженности эндоморфизма $\psi$.

7) Первое равенство немедленно следует из п. 2) предложения 1. Пусть теперь $b \in \operatorname{ker} \psi$. Тогда в силу (25) $b \diamond e_{a}=0, a=1, \ldots, n$. Более того, в силу п. 5) предложения $1 b \diamond f\left(e_{a}\right)=-f\left(b \diamond e_{a}\right)=0$. Следовательно, $b \diamond u=0 \quad(u \in \mathscr{L})$, и, значит, $\operatorname{ker} \psi \diamond \mathscr{L}=\mathscr{L} \diamond \operatorname{ker} \psi=\{0\}$.

8) Пусть $b \in \mathscr{L} \diamond \mathscr{L}$. Тогда $b=\sum_{k=1}^{N} u_{k} \diamond v_{k}$, где $u_{k}, v_{k} \in \mathscr{L}$. Пусть $c \in \operatorname{ker} \psi$. Тогда в силу (18) и п. 7)

$$
\langle c, b\rangle=\sum_{k=1}^{N}\left\langle c, u_{k} \diamond v_{k}\right\rangle=-\sum_{k=1}^{N}\left\langle u_{k} \diamond c, v_{k}\right\rangle=0 .
$$

Следовательно, $\mathscr{L} \diamond \mathscr{L} \perp \operatorname{ker} \psi$. Но тогда в силу п. 6) $\mathscr{L} \diamond \mathscr{L} \subset \operatorname{Im} \psi$. Обратно: если $c \in \operatorname{Im} \psi$, то в силу (23) и предложения 1

$$
c=\psi(b)=-\sum_{a}\left(b \diamond e_{a}\right) \diamond e_{a} \in \mathscr{L} \diamond \mathscr{L} .
$$

Следовательно, $\mathscr{L} \diamond \mathscr{L}=\operatorname{Im} \psi$.

ЗАмЕчАниЕ. Из пп. 1), 3) легко следует, что естественно определен комплексный эндоморфизм $\varphi \in$ End $\mathscr{M}^{c}$ такой, что $\varphi+\bar{\varphi}=\psi$. Именно,

$$
\varphi(X)=-\frac{1}{2} \sum_{a=1}^{n}\left(X \diamond \varepsilon^{a}\right) \diamond \varepsilon_{a}, \quad X \in \mathscr{M}^{c} .
$$

Этот эндоморфизм, очевидно, не зависит от выбора $A$-репера и имеет в произвольном $A$-репере матрицу $\left(\varphi_{\beta}^{\alpha}\right)$, где $\varphi_{\beta}^{\alpha}=(1 / 4) B_{h c}^{\alpha} B_{\beta}^{h c}$.

Напомним [10], что на метрическом $f$-многообразии, рассматриваемом как обобщенное почти эрмитово многообразие, внутренним образом определен самосопряженный эндоморфизм $\alpha(X)=\sum_{a=1}^{n} A\left(X, e_{a}, e_{a}\right)$ присоединенной $K$-алгебры, где $A$ - тензор кривизны присоединенной $K$-алгебры с компонентами $A_{b c}^{a d}=$ $A\left(\varepsilon_{b}, \varepsilon_{c}, \varepsilon_{\hat{d}}\right), A_{\hat{b} \hat{c}}^{\hat{a} \hat{d}}=\bar{A}_{b c}^{a d}$ на пространстве присоединенной $G$-структуры. Очевидно, распределения $\mathscr{L}$ и $\mathscr{M}$ инвариантны относительно $\alpha$, причем сужение $\alpha$ на $\mathscr{M}$ нулевое, и поэтому $\alpha$ можно рассматривать как элемент кольца End $\mathscr{L}$. Очевидно, в $A$-репере этот эндоморфизм задается матрицей $\left(\begin{array}{cc}\alpha_{c}^{b} & 0 \\ 0 & \alpha_{\hat{c}}^{\hat{b}}\end{array}\right)$, где $\alpha_{c}^{b}=A_{c h}^{b h}, \alpha_{\hat{c}}^{\hat{b}}=\bar{\alpha}_{c}^{b}=\alpha_{b}^{c}$.

ОПРЕДЕЛЕНИЕ 1. Эндоморфизмы $\theta, \psi, \alpha$ назовем каноническими әндоморфизмами первого, второго и третьего рода соответственно.

ОпРедЕЛЕниЕ 2. Распределение на $f$-многообразии назьвается голоморфным.м, если оно инвариантно относительно действия структурного эндоморфизма. 
ОПРЕДЕЛЕНИЕ 3 . Пусть $\left(M_{1}, f_{1}, g_{1}\right)$ и $\left(M_{2}, f_{2}, g_{2}\right)$ - два метрических $f$-многообразия. Диффеоморфизм $\phi: M_{1} \rightarrow M_{2}$ называется әквивалентностью, если: 1) $\phi$-изометрия, т.е. $\left.\phi^{*} g_{2}=g_{1} ; 2\right) \phi \circ f_{1}=f_{2} \circ \phi$.

Введем в рассмотрение на киллинговом $f$-многообразии $M$ распределения $\mathscr{D}_{P}=$ $\operatorname{Im} \theta \oplus \operatorname{Im} \psi, \mathscr{D}_{N}=\operatorname{ker} \theta$ и $\mathscr{D}_{R}=\operatorname{ker} \psi$.

Tеорема 3. Распределения $\mathscr{D}_{P}, \mathscr{D}_{N}$ и $\mathscr{D}_{R}$ являются голоморфными идеалами канонической $Q$-алгебры $(\mathscr{W}, \bullet)$ многообразия $M$, причем $\mathscr{W}$ распадается в их прямое произведение.

ДокАЗАТЕЛЬСТво. Прежде всего заметим, что в силу п. 6) теоремы 1 и п. 6) теоремы $2 \mathrm{X}(M)=\mathscr{D}_{N} \oplus \mathscr{D}_{P} \oplus \mathscr{D}_{R}$, причем компоненты этой прямой суммы попарно ортогональны. Покажем, что $\mathscr{D}_{P} \subset(\mathscr{W}, \bullet)$ - идеал. Пусть $X \in \mathscr{W}, A \in \mathscr{D}_{P}$. Тогда $X=u+b, A=v+c$, где $u \in \mathscr{L}, b \in \mathscr{M}, v \in \operatorname{Im} \theta, c \in \operatorname{Im} \psi$. Поскольку $\mathscr{M}$ входит в аннулятор присоединенной $K$-алгебры [9], $\mathscr{M} * W=W * \mathscr{M}=\{0\}$. Кроме того, в силу п. 4) предложения $1 \mathscr{M} \diamond \mathscr{M}=\{0\}$, и, значит, $\mathscr{M} \bullet \mathscr{M}=\{0\}$. Поэтому $X \bullet A=u * v+u \diamond v+b \diamond v+u \diamond c$. Далее, так как $v \in \operatorname{Im} \theta$, в силу п. 8) теоремы 1

$$
v=\sum_{k=1}^{N} a_{k} \diamond u_{k}, \quad a_{k} \in \mathscr{M}, \quad u_{k} \in \mathscr{L} .
$$

Следовательно,

$$
u * v=u *\left(\sum_{k=1}^{N} a_{k} \diamond u_{k}\right)=\sum_{k=1}^{N} u *\left(a_{k} \diamond u_{k}\right)=0
$$

в силу п. 7) предложения 1 . Далее, $u \diamond v \in \operatorname{Im} \psi$ в силу п. 8) теоремы 2, a $b \diamond v \in \operatorname{Im} \theta$ и $u \diamond c \in \operatorname{Im} \theta$ в силу п. 8) теоремы 1 . Следовательно, $X \bullet A \in \mathscr{D}_{P}$. Аналогично, $A \bullet X \in \mathscr{D}_{P}$, т.е. $\mathscr{D}_{P} \subset \mathscr{W}$ - двусторонний идеал.

Покажем, что $\mathscr{D}_{R} \subset \mathscr{W}$-идеал. Пусть $X \in \mathscr{W}, b \in \mathscr{D}_{R}$. Тогда $X=u+a, u \in \mathscr{L}$, $a \in \mathscr{M}$, и в силу того, что $\mathscr{M} \bullet \mathscr{M}=\{0\}$, a $\mathscr{D}_{R} \subset \mathscr{M}, X \bullet b=u \bullet b=u * b+u \diamond b=u \diamond$ $b=0$ в силу п. 7) теоремы 2. Следовательно, $X \bullet b=0$. Аналогично, $b \bullet X=0$. В частности, $\mathscr{D}_{R} \subset \mathscr{W}$ - двусторонний абелев идеал. Но тогда $\mathscr{D}_{P} \oplus \mathscr{D}_{R} \subset \mathscr{W}-$ идеал, а значит, $\mathscr{D}_{N} \subset \mathscr{W}$ - идеал как его ортогональное дополнение. Заметим, что эти идеалы, очевидно, являются также и идеалами $Q$-алгебры $(\mathscr{W}, \diamond)$.

Докажем, что $\mathscr{D}_{P}$ - голоморфное распределение. Пусть $X \in \mathscr{D}_{P}$. Тогда $X=$ $\theta(u)+\psi(a)$ для некоторых $u \in \mathscr{L}, a \in \mathscr{M}$. Но тогда в силу п. 4) теоремы 1 и п. 4) теоремы $2 f(X)=f \circ \theta(u)=\theta \circ f(u) \in \operatorname{Im} \theta \subset \mathscr{D}_{P}$. Значит, $f\left(\mathscr{D}_{P}\right) \subset \mathscr{D}_{P}$. Далее, пусть $X \in \mathscr{D}_{N}$, т.е. $\theta(X)=0$. Но тогда в силу п. 4) теоремы $1 \theta(f X)=f \circ \theta X=0$, т.е. $f(X) \in \mathscr{D}_{N}$. Следовательно, $f\left(\mathscr{D}_{N}\right) \subset \mathscr{D}_{N}$. Аналогично, $f\left(\mathscr{D}_{R}\right) \subset \mathscr{D}_{R}$.

Заметим, что тройка $\{g=\langle\cdot, \cdot\rangle, f, T\}$ в силу $\left(11_{3,4}\right)$ и (15) определяет на $M$ структуру квазиоднородного многообразия относительно связности $\widehat{\nabla}$ в том смысле, что $\widehat{\nabla} g=0, \widehat{\nabla} f=0, \widehat{\nabla} T=0$. Отсюда теми же рассуждениями, что и при доказательстве теоремы 13 из [9], убеждаемся в том, что разложение $Q$-алгебры $\mathscr{W}$ 
в прямое произведение идеалов $\mathscr{D}_{N}, \mathscr{D}_{P}$ и $\mathscr{D}_{R}$ влечет разложение касательного пучка многообразия $M$ в ортогональную прямую сумму $f$-инвариантных распределений, инвариантных относительно действия группы голономии римановой связности, причем тензоры $g, \Omega$ и $T$ будут разложимыми, а многообразие $M$ локально эквивалентно произведению киллинговых $f$-многообразий $M_{N}, M_{P}$ и $M_{R}$, которые можно интерпретировать как вполне геодезические интегральные многообразия распределений $\mathscr{D}_{N}, \mathscr{D}_{P}$ и $\mathscr{D}_{R}$ соответственно. Очевидно, $M_{N}-$ приближенно келерово многообразие (поскольку $\mathscr{D}_{N} \subset \mathscr{L}$, и, значит, сужение $f$ на $\mathscr{D}_{N}$ невырождено), $M_{R}$ - риманово многообразие без дополнительной структуры (поскольку $\mathscr{D}_{R} \subset \mathscr{M}$, и, значит, сужение $f$ на $\mathscr{D}_{R}$ тождественно равно нулю). Наконец, особый интерес представляет многообразие $M_{P}$, которое характеризуется тем, что для него $\operatorname{ker} \theta=\{0\}, \operatorname{ker} \psi=\{0\}$, т.е. эндоморфизмы $\theta$ и $\psi$ невырожденные, а значит, $\operatorname{Im} \theta=\mathscr{L}, \operatorname{Im} \psi=\mathscr{M}$. Согласно п. 8) теоремы 1 и п. 8) теоремы 2 эти условия можно записать в форме

$$
\mathscr{L} \diamond \mathscr{M}=\mathscr{M} \diamond \mathscr{L}=\mathscr{L}, \quad \mathscr{L} \diamond \mathscr{L}=\mathscr{M}
$$

ОПРЕДЕЛЕНИЕ 4. Киллингово $f$-многообразие называется собственно киллинговым , если его фундаментальные распределения удовлетворяют соотношениям (26), или, что равносильно, если его канонические эндоморфизмы первого и второго рода невырождены.

Таким образом, мы получаем следующий фундаментальный результат.

ТЕОРЕмА 4. Всякое киллингово $f$-многообразие локально әквивалентно произведенил приближенно келерова многообразия, собственно киллингова f-многообразия и риманова многообразия без дополнительной структуры.

Заметим, что этот результат впервые был анонсирован в [4]. Из этой теоремы следует, в частности, что неприводимое киллингово $f$-многообразие $M$ дефекта $0<\operatorname{def} M<\operatorname{dim} M$ является собственно киллинговым $f$-многообразием. Примером таких многообразий является пятимерная сфера $S^{5}$, наделенная канонической слабо косимплектической структурой, индуцированной при ее вложении в классическое приближенно келерово многообразие $S^{6} \subset \mathbf{O}$ в качестве вполне геодезического подмногообразия [5].

Следуя терминологии теории обобщенных почти контактных многообразий [12], назовем метрическое $f$-многообразие многообразием келерова типа, если его присоединенная $Q$-алгебра абелева.

ТЕОРЕМа 5. Собственно киллингово $f$-многообразие является многообразием келерова типа.

ДокАЗАТЕЛЬСтво. По существу это уже было доказано при доказательстве теоремы 3. Приведем прямое рассуждение. Пусть $M-$ собственно киллингово $f$-многообразие, $X, Y \in \mathrm{X}(M)$. Тогда $X * Y \in \mathscr{L}$ (см. [9]), и в силу п. 1) теоремы 1 и п. 7) предложения $1 \theta(X * Y)=-\sum_{a}\left(\left((X * Y) \diamond \varepsilon_{\alpha}\right) \diamond \varepsilon_{\alpha}\right)=0$. Из невырожденности эндоморфизма $\theta$ отсюда следует, что $X * Y=0$, т.е. $\mathscr{W} * \mathscr{W}=0$. 
ЗАмЕчАниЕ. Согласно формуле (19) ненулевые компоненты композищионного тензора присоединенной $K$-алгебры вычисляются по формулам $\mathscr{T}_{\hat{b} \hat{c}}^{a}=B^{a b c}$ и по их комплексньм сопряжениям. В силу этого теорема 5 означает, что структурные тензоры первого рода собственно киллингова $f$-многообразия нулевые, что сушественно упрошает вид структурных уравнений (7) и (9), а также соотношений (12)-(14).

Пусть $M$ - киллингово $f$-многообразие. Рассмотрим семейство $\mathscr{B}=\left\{B_{Z}=\right.$ $\left.L_{\mathrm{m} Z} \mid Z \in \mathrm{X}(M)\right\} \subset$ End $\mathscr{L}$ эндоморфизмов модуля $\mathscr{L}$. В силу (18) эндоморфизмы этого семейства кососимметричны:

$$
\left\langle B_{Z}(X), Y\right\rangle+\left\langle X, B_{Z}(Y)\right\rangle=0, \quad X, Y \in \mathscr{L} .
$$

ОПРЕДЕЛЕНИЕ 5 . Киллингову $f$-структуру на $M$ назовем коммутативнокиллинговой, если $\mathscr{B}$ - коммутативное семейство, т.е.

$$
B_{X} \circ B_{Y}=B_{Y} \circ B_{X}, \quad X, Y \in \mathrm{X}(M) .
$$

Собственно коммутативно-киллингову $f$-структуру назовем $C$-киллинговой структурой.

Очевидно, $C$-киллинговыми структурами являются все собственные слабо косимплектические структуры, поскольку их дефект равен 1.

ЗАмЕчАнИЕ. Прямым подсчетом с учетом $\left(12_{2}\right)$ и (14) проверяется, что условие (28) равносильно тождеству $W(\mathrm{~m} X, \mathrm{~m} Y) \mathrm{I} Z=0, X, Y, Z \in \mathrm{X}(M)$, где $W$ тензор Вейля конформной кривизны, в частности, это условие конформно-инвариантно.

Пусть $M$ - собственно киллингово $f$-многообразие. Дифференциальное продолжение второй группы структурных уравнений - уравнений (9) - приводит нас к тождествам:

$$
\begin{aligned}
& \text { 1) } A_{b h}^{a[g} B_{\beta}^{f] h}+\frac{3}{2} B_{b h}^{\alpha} B_{\alpha}^{a[g} B_{\beta}^{f] h}+3 B_{[\alpha}^{a h} B_{\beta] h b} B^{\alpha f g}=0 ; \\
& \text { 2) } A_{\beta \gamma \delta}^{\alpha} B^{\gamma f g}+\frac{3}{2}\left(B_{r h}^{\alpha} B_{\beta}^{h[g} B_{\delta}^{f] r}-B_{\beta r h} B^{\alpha h[g} B_{\delta}^{f] r}\right)=0 .
\end{aligned}
$$

Заметим, что $\left(29_{2}\right)$ можно переписать в форме

$$
A_{\beta \gamma \delta}^{\alpha} B^{\gamma f g}+3 B_{r h}^{[\alpha} B^{\beta] h[g} B_{\delta}^{f] r}=0 .
$$

В частности, если $M-C$-киллингово многообразие, то $A_{\beta \gamma \delta}^{\alpha} B^{\gamma f g}=0$. Свертьвая это соотношение с $B_{\gamma f c}$, в силу п. 3 ) теоремы 1 и невырожденности эндоморфизма $\theta$ получим, что $A_{\beta \gamma \delta}^{\alpha}=0$. Доказано

ПрЕДЛОЖЕНИЕ 2. Структурный тензор четвертого рода С-киллингова многообразия тождественно равен нулю.

Более того, заметим, что распределение $\mathscr{M}$ на пространстве присоединенной $G$-структуры произвольного киллингова $f$-многообразия задается системой Пфаффа $\omega^{a}=0, \omega_{a}=0$, которая в силу (7) вполне интегрируема, а в силу соотношений $\left(6_{3-6}\right)$ максимальные интегральные многообразия этой системы вполне 
геодезичны. Если к тому же $M-C$-киллингово многообразие, то в силу $\left(12_{3}\right)$ и предложения 2 они плоски. Обратно: если второе фундаментальное распределение собственно киллингова $f$-многообразия плоско (т.е. все его интегральные многообразия плоски), то структурный тензор четвертого рода этого многообразия равен нулю, и в силу $\left(29_{2}\right)$

$$
B_{r h}^{[\alpha} B_{h g}^{\beta]} B_{\delta}^{f r}=B_{r h}^{[\alpha} B_{h f}^{\beta]} B_{\delta}^{g r} .
$$

Свертывая это соотношение с $B_{r c}^{\delta}$, с учетом п. 3 ) теоремы 1 получим $C_{b}^{a} \theta_{c}^{d}=C_{b}^{d} \theta_{c}^{a}$, где $C=B_{X} \circ B_{Y}-B_{Y} \circ B_{X}, X, Y \in \mathrm{X}(M)$. Свернем это соотношение по индексам $d$ и $b: C \circ \theta=(\operatorname{tr} C) \theta$. Поскольку эндоморфизм $C$, очевидно, бесследен, а эндоморфизм $\theta$ обратим, отсюда следует, что $C=0$, т.е. $M-C$-киллингово многообразие. Доказана

Теорема 6. Пусть $M$ - собственно киллингово $f$-многообразие. Тогда следующие условия әквивалентны:

1) $M-C$-киллингово многообразие;

2) структурный тензор четвертого рода многообразия $M$ равен нулю;

3) второе фундаментальное распределение многообразия $M$ плоско.

Далее, если $M-C$-киллингово многообразие, соотношение $\left(29_{1}\right)$ перепишется в виде

$$
2 A_{b h}^{a g} B_{\beta}^{f h}-2 A_{b h}^{a f} B_{\beta}^{g h}+3 B_{b h}^{\alpha} B_{\alpha}^{a g} B_{\beta}^{f h}-3 B_{b h}^{\alpha} B_{\alpha}^{a f} B_{\beta}^{g h}=0 .
$$

Свернем это соотношение по индексам $b$ и $f$ с учетом п. 3$)$ теоремы 1 и $\left(10_{1}\right)$ :

$$
-2 \alpha_{h}^{a} B_{\beta}^{g h}+3 B_{b h}^{\alpha} B_{\beta}^{b h} B_{\alpha}^{a g}+3 \theta_{h}^{a} B_{\beta}^{g h}=0
$$

Свернем обе части этого соотношения с $Z^{\beta} X_{g}, X \in \mathscr{L}, Z \in \mathscr{M}$. Ввиду (19) и замечания к теореме 2 получим

$$
2\left(\alpha \circ B_{Z}\right) X+12 B_{\varphi(Z)} X-3\left(\theta \circ B_{Z}\right) X=0 .
$$

Таким образом,

$$
(2 \alpha-3 \theta) \circ B_{Z}=-12 B_{\varphi(Z)} .
$$

Далее, с учетом (27) и самосопряженности эндоморфизмов $\alpha$ и $\theta$, вводя обозначение $\widehat{\alpha}=2 \alpha-3 \theta$, получим из (31)

$$
12\left\langle B_{\varphi(Z)} X, Y\right\rangle=-12\left\langle X, B_{\varphi(Z)} Y\right\rangle=\left\langle X,\left(\widehat{\alpha} \circ B_{Z}\right) Y\right\rangle=-\left\langle\left(B_{Z} \circ \widehat{\alpha}\right) X, Y\right\rangle,
$$

и в силу невырожденности метрики $B_{Z} \circ \widehat{\alpha}=-12 B_{\varphi(Z)}$. В частности, $\widehat{\alpha} \circ B_{Z}=$ $B_{Z} \circ \widehat{\alpha}$, откуда

$$
2 B_{Z} \circ \alpha=2 \alpha \circ B_{Z}-3 \theta \circ B_{Z}+3 B_{Z} \circ \theta .
$$

Аналогично, свертывая (30) по индексам $a$ и $b$, получим

$$
B_{Z} \circ(2 \alpha+3 \theta)+(2 \alpha+3 \theta) \circ B_{Z}=0 .
$$


Сравнивая это соотношение с (32), получаем

$$
2 \alpha \circ B_{Z}=-3 B_{Z} \circ \theta, \quad 2 B_{Z} \circ \alpha=-3 \theta \circ B_{Z} .
$$

Подставляя эти соотношения в (31), получаем:

1) $B_{Z} \circ \theta+\theta \circ B_{Z}=4 B_{\varphi(Z)}$;

2) $B_{Z} \circ \alpha+\alpha \circ B_{Z}=-6 B_{\varphi(Z)}$;

где $Z \in \mathscr{M}$

ОПРЕДЕЛЕНИЕ 6. $C$-киллингову структуру назовем скалярной (короче: $S C$ структурой), если ее канонический эндоморфизм третьего рода скалярен, т.е. $\alpha=\lambda \mathrm{id}$

Пусть $M-S C$-многообразие. Тогда в силу $(33) B_{Z}(3 \theta+2 \lambda \mathrm{id})=0$, и, следовательно, $(3 \theta+2 \lambda \mathrm{id}) X \circ(\mathrm{m} Y)=0, X \in \mathscr{L}, Y \in \mathrm{X}(M)$. Поэтому

$$
\theta((3 \theta+2 \lambda \mathrm{id})(X))=-\sum_{\alpha}\left((3 \theta+2 \lambda \mathrm{id})(X) \diamond \varepsilon_{\alpha}\right) \diamond \varepsilon_{\alpha}=0
$$

и в силу невырожденности эндоморфизма $\theta$

$$
\theta=-\frac{2}{3} \lambda \mathrm{id}
$$

Но тогда в силу $\left(34_{1}\right) \quad \lambda B_{Z}=-3 B_{\varphi(Z)}$, и, значит, $((3 \varphi+\lambda \mathrm{id}) \mathrm{m} Z) \diamond X=0$. Поэтому $\sum_{a}\left(((3 \varphi+\lambda \mathrm{id}) \mathrm{m} Z) \diamond e_{a}\right) \diamond e_{a}=0$, т.е. $\psi(3 \varphi+\lambda \mathrm{id})(\mathrm{m} Z)=0$, и в силу невырожденности эндоморфизма $\psi$ имеем $\varphi=-(1 / 3) \lambda \mathrm{id}$. Более того, поскольку $\alpha$-вешественный эндоморфизм, то $\lambda \in \mathbb{R}$, значит,

$$
\psi=-\frac{2}{3} \lambda \mathrm{id}
$$

Доказано

ПРЕДЛОЖЕНИЕ 3. Все канонические әндоморфизмы $S C$-многообразия скалярны.

Заметим, что согласно п. 3) теоремы 1

$$
\operatorname{tr} \theta=2 B^{\alpha b c} B_{\alpha b c}=2 \sum_{\alpha, b, c}\left|B_{\alpha b c}\right|^{2} .
$$

С другой стороны, согласно $(35) \operatorname{tr} \theta=-(4 / 3) n \lambda$, и, значит,

$$
\lambda=-\frac{3}{2 n} \sum_{\alpha, b, c}\left|B_{\alpha b c}\right|^{2} .
$$

Аналогично, согласно п. 3) теоремы 2

$$
\operatorname{tr} \psi=\frac{1}{2} B^{\alpha b c} B_{\alpha b c}=\frac{1}{2} \sum_{\alpha, b, c}\left|B_{\alpha b c}\right|^{2} .
$$


С другой стороны, согласно (36) $\operatorname{tr} \psi=-(2 / 3) m \lambda$, и, значит,

$$
\lambda=-\frac{3}{4 m} \sum_{\alpha, b, c}\left|B_{\alpha b c}\right|^{2} .
$$

Сравнивая эти соотношения, находим, что $n=2 m$, и, значит, $\operatorname{dim} M=5 m$. Отметим, кроме того, что $\lambda<0$. Далее, с учетом (14), (35), п. 3 ) теоремы 1 и п. 3 ) теоремы 2 имеем в $A$-репере

$$
\begin{aligned}
r_{b}^{a} & =\frac{5}{2} \theta_{b}^{a}-\alpha_{b}^{a}=-\frac{5}{3} \lambda \delta_{b}^{a}-\lambda \delta_{b}^{a}=-\frac{8}{5} \lambda \delta_{b}^{a}, \\
r_{\beta}^{\alpha} & =r_{\alpha \beta}=B_{\alpha}^{d h} B_{\beta d h}+B_{\beta}^{d h} B_{\alpha d h} \\
& =B^{\alpha d h} B_{\beta d h}+B^{\alpha d h} B^{\beta d h}=4 \psi \delta_{\beta}^{a}=-\frac{8}{3} \lambda \delta_{\beta}^{\alpha}, \\
r_{a b} & =r_{\hat{a} \hat{b}}=r_{\alpha \beta}=0 .
\end{aligned}
$$

Следовательно,

$$
r=-\frac{8}{3} \lambda \mathrm{id}=\left(\frac{2}{m} \sum_{\alpha, b, c}\left|B_{\alpha b c}\right|^{2}\right) \mathrm{id}
$$

Доказана

ТеОРема 7. SC-многообразие дефекта $m$ является 5 т-мерныцм многообразием Эйнштейна с космологической константой $\varepsilon=(2 / m) \sum_{\alpha, b, c}\left|B_{\alpha b c}\right|^{2}$.

В частности, кривизна Риччи $S C$-многообразия положительна и отделена от нуля. С учетом известной теоремы Майерса [13] получаем

СлеДСТВИЕ. Полное $S C$-многообразие компактно и имеет конечную фундаментальную группу.

ПримеР. Пусть $M^{2 n+m}-C$-киллингово многообразие точечно постоянной голоморфной секционной кривизны $2 c$ (т.е. кривизны в двумерных направлениях вида $X \wedge f X, X \in \mathscr{L})$. Из (17) непосредственно следует, что оно является обобщенным квазикелеровым многообразием [10], и в силу теоремы 24 из [10] его присоединенная $K$-алгебра тривиально оснащена, а именно,

$$
A(X, Y, Z)=-\frac{c}{4}(\langle\langle X, Z\rangle\rangle Y+\langle\langle Y, Z\rangle\rangle X)
$$

где $\langle\langle X, Y\rangle\rangle=\langle X, Y\rangle+\sqrt{-1}\langle X, f Y\rangle$ - метрика присоединенной $K$-алгебры. Отсюда следует, что $\alpha=-((n+1) / 4) c \mathrm{id}$, и в силу $(35)$ и $(36) \theta=\psi=((n+1) / 6) c$ id.

С учетом теоремы 7 и ее следствия получается

ТЕОрема 8. С-киллингово многообразие дефекта $m$ точечно постоянной $f$-голоморфной секционной кривизны с является 5 -мерным многообразием Эйнштейна с космологической константой $\varepsilon=(2 / 3)(2 m+1) c ; f$-голоморфная секционная кривизна такого многообразия глобально постоянна и положительна. В случае полноты такое многообразие компактно и имеет конечную фундаментальную группу. 
Напомним, что согласно А. Грею [1] приближенно келерово многообразие $(M, J,\langle\cdot, \cdot\rangle)$ называется многообразием постоянного типа $c$, если

$$
\forall X, Y \in \mathrm{X}(\mathrm{M}) \&\langle X, Y\rangle=\langle X, J Y\rangle=0 \Rightarrow\left\|\nabla_{X}(J) Y\right\|^{2}=c\|X\|^{2}\|Y\|^{2} .
$$

Обобшая это определение, примем следующее

ОПРЕДЕЛЕНИЕ 7 . Киллингово $f$-многообразие $M$ назовем многообразием постоянного типа с, если

$$
\forall X, Y \in \mathscr{L} \&\langle\langle X, Y\rangle\rangle=0 \Rightarrow\|X \bullet Y\|^{2}=c\|X\|^{2}\|Y\|^{2} .
$$

Заметим, что в случае приближенно келерова многообразия эти определения в силу (17) совпадают, поскольку для такого многообразия структурньй эндоморфизм $f$ обратим, а $S=\mathscr{T}$.

Пусть $M$ - коммутативно-киллингово $f$-многообразие постоянного типа $c \neq 0$. Очевидно, $M$ в этом случае голоморфно неприводимо, т.е. локально неэквивалентно произведению $f$-многообразий меньшей размерности. В силу теоремы $4 M$ в этом случае является либо приближенно келеровым, либо $C$-киллинговым многообразием. Поскольку первый случай полностью изучен [7], будем предполагать, что $M$ является $C$-киллинговым многообразием. Пусть $X, Z \in \mathscr{L}$ - произвольная пара векторов. Пусть $X \neq 0$. Тогда вектор $Z$ можно представить в виде $Z=\lambda X+\mu f(X)+Y$, где $\langle X, Y\rangle=\langle X, f Y\rangle=0$, т.е. $\langle\langle X, Y\rangle\rangle=0$. При этом $\lambda=\|X\|^{-2}\langle Z, X\rangle, \mu=\|X\|^{-2}\langle Z, f(X)\rangle$. С учетом этих соотношений, а также поскольку в силу (19) $X \diamond X=0, X \diamond f(X)=f(X) \diamond X=-X \diamond f(X)$, то

$$
\begin{gathered}
X \diamond f(X)=0, \\
\|X \diamond Z\|^{2}=\|X \diamond(\lambda X+\mu f(X)+Y)\|^{2}=\|X \diamond Y\|^{2}=c\|X\|^{2}\|Y\|^{2} \\
=c\|X\|^{2}\|Z-\lambda X-\mu f(X)\|^{2},
\end{gathered}
$$

и после очевидных преобразований получаем

$$
\|X \diamond Z\|^{2}=c\left(\|X\|^{2}\|Z\|^{2}-\langle Z, X\rangle^{2}-\langle Z, f(X)\rangle^{2}\right) .
$$

Поляризуем это соотношение, заменяя $X$ на $X+Y(X, Y \in \mathscr{L})$ и замечая, что в силу (18) и (19) $\langle X \diamond Z, Y \diamond Z\rangle=-\langle Z \diamond(Z \diamond Y), X\rangle$ :

$$
\langle Z \diamond(Z \diamond Y), X\rangle=-c\left\{\|Z\|^{2}\langle Y, X\rangle-\langle Z, Y\rangle\langle Z, X\rangle-\langle Z, f(Y)\rangle\langle Z, f(X)\rangle\right\}
$$

Заменив в этом соотношении $X$ на $f(X)$ и почленно прибавив полученное соотношение, умноженное на $\sqrt{-1}$, к $(39)$, получим

$$
\langle\langle Z \diamond(Z \diamond Y), X\rangle\rangle=-c\left\{\|Z\|^{2}\langle\langle Y, X\rangle\rangle-\langle\langle Y, Z\rangle\rangle\langle\langle Z, X\rangle\rangle\right\} .
$$


В силу невырожденности метрики отсюда следует

$$
Z \diamond(Z \diamond Y)=-c\left\{\|Z\|^{2} Y-\langle\langle Y, Z\rangle\rangle Z\right\}
$$

Заметим, что в силу (19) тернарная операция $[X, Y, Z]=X \diamond(Y \diamond Z)$ в распределении $\mathscr{L}$, рассматриваемом как модуль над кольцом гладких комплекснозначных функций на $M$, антилинейна по первому и линейна по двум остальньм аргументам. Инволюционно поляризуя (см. [12]) с учетом этого соотношение (40), получим

$$
X \diamond(Y \diamond Z)=c\{\langle\langle Z, X\rangle\rangle Y-\langle\langle Y, X\rangle\rangle Z\}, \quad X, Y, Z \in \mathscr{L}
$$

Очевидно, что верно и обратное: $C$-киллингово многообразие, для которого справедливо тождество (41), имеет постоянный тип $c$. Более того, это тождество с учетом (19) и того обстоятельства, что в $A$-репере $\left\langle\left\langle\varepsilon_{\hat{a}}, \varepsilon_{b}\right\rangle\right\rangle=\delta_{b}^{a}$, на пространстве присоединенной $G$-структуры запишется в форме $B_{\alpha}^{a d} B_{b c}^{\alpha}=2 c \delta_{b c}^{a d}$, где $\delta_{b c}^{a d}=$ $\delta_{b}^{a} \delta_{c}^{d}-\delta_{c}^{a} \delta_{b}^{d}$

Доказана

ТЕОРема 9. Пусть $M-C$-киллингово многообразие. Тогда следующие утверждения эквивалентны:

1) $M$ - многообразие постоянного типа с;

2) $X \diamond(Y \diamond Z)=c\{\langle\langle Z, X\rangle\rangle Y-\langle\langle Y, X\rangle\rangle Z\}, \quad X, Y, Z \in \mathscr{L}$;

3) на пространстве присоединенной $G$-структурь $B_{\alpha}^{a d} B_{b c}^{\alpha}=2 c \delta_{b c}^{a d}$.

Пусть $M-C$-киллингово многообразие. Свернем обе части соотношения $(30)$ с тензором $B_{f r}^{\beta}$ :

$$
2 A_{b h}^{a g} B_{f r}^{f h}-2 A_{b h}^{a f} B_{f r}^{g h}+3 B_{b h}^{a g} B_{f r}^{f h}-3 B_{b h}^{a f} B_{f r}^{g h}=0,
$$

где $B_{b c}^{a d}=B_{\alpha}^{a d} B_{c d}^{\alpha}$. В частности, если $M$ - многообразие постоянного типа $c$, то $B_{b c}^{a d}=2 c \delta_{b c}^{a d}$, и после соответствующих преобразваний (42) примет вид

$$
(n-2) A_{b r}^{a g}+\alpha_{b}^{a} \delta_{r}^{g}=3 c\left\{(3-2 n) \delta_{b}^{a} \delta_{r}^{g}+(n-2) \delta_{r}^{a} \delta_{b}^{g}\right\}
$$

Свернем это соотношение по индексам $g$ и $r$, заметив, что в силу п. 3 ) предложения 1 и $(26) n \neq 1$ :

$$
\alpha_{b}^{a}=-3 c(n-1) \delta_{b}^{a} .
$$

Подставим это соотношение в (43):

$$
(n-2) A_{b r}^{a g}=3 c\left\{(2-n) \delta_{b}^{a} \delta_{r}^{g}+(n-2) \delta_{r}^{a} \delta_{b}^{g}\right\}
$$

Если $n \neq 2$, то отсюда следует, что $A_{b r}^{a g}=-3 c \delta_{b r}^{a g}$. Альтернируя это соотношение по индексам $b$ и $r$, с учетом $\left(10_{1}\right)$ получаем $c=0$, что противоречит предположению. Следовательно, $n=2$, причем выполняется соотношение (44). Но тогда $M$ является $S C$-многообразием, и в силу теоремы $7 \operatorname{dim} M=4+m=5 m$, т.е. $\operatorname{def} M=1$ и $\operatorname{dim} M=5$. 
Обратно: пусть $M$ - пятимерное $S C$-многообразие. Тогда из условия $n=2$ вытекает, что $B_{a b}^{\alpha}=\xi^{\alpha} B_{a b}$, где $\xi \in \mathscr{M}$ - единичный вектор, $\left\{B_{a b}\right\}$ - компоненты комплексно-линейной 2-формы на распределении $\mathscr{L}$, рассматриваемом как модуль над кольцом гладких комплекснозначных функций на $M$. Действительно, каждый вектор $U \in \mathscr{M}$ определяет отображение $C_{U}: \mathscr{L} \otimes \mathscr{L} \rightarrow C^{\infty}(M)$ по формуле $C_{U}(X, Y)=B(U, X, Y)-\sqrt{-1} B(U, X, f Y)$, где $B(U, X, Y)=B_{\alpha b c} U^{\alpha} X^{b} Y^{c}+$ $B^{\alpha b c} U_{\alpha} X_{b} Y_{c}$. Очевидно, $C_{U}$ - комплексно-линейная 2-форма на $\mathscr{L}$, и поскольку $\operatorname{dim}_{C} \mathscr{L}=2, C_{U}(X, Y)=f(U) \tau(X, Y)$, где $f$ - ковектор, то $\tau$ - базисная 2-форма. Следовательно, $B=f \otimes \tau$, или (если рассматривать $B$ как отображение $B: \mathscr{L} \times \mathscr{L} \rightarrow \mathscr{M}$, т.е. поднять индекс типа $\mathscr{M}) B=\xi \otimes \tau$, т.е. $B_{a b}^{\alpha}=B_{a b} \xi^{\alpha}$, $B_{\alpha}^{a b}=B^{a b} \xi_{\alpha}$. Очевидно, перенормируя в случае необходимости $\xi$ и $\tau$, без ограничения обшности можно считать, что $\|\xi\|=1$. При этом $B^{c d} B_{a b}=c \delta_{a b}^{c d}$ для некоторого $c \in C^{\infty}(M)$, т.е. многообразие $M$ имеет ненулевой постоянный тип. Таким образом, класс $C$-киллинговых многообразий ненулевого постоянного типа совпадает с классом пятимерных $S C$-многообразий. Согласно (44) в этом случае $\alpha=-3 c$ id. Более того, дифференциальное продолжение соотношений $\left(9_{1}\right)$ показывает, что в этом случае $A_{b c}^{a d}=-c \tilde{\delta}_{b c}^{a d}$, где $\tilde{\delta}_{b c}^{a d}=\delta_{b}^{a} \delta_{c}^{d}+\delta_{c}^{a} \delta_{b}^{d}$. С учетом соотношений $(12),(13)$ и теоремы 9 получаем отсюда, что $R_{\hat{a} b c \hat{d}}=2 c \delta_{b}^{a} \delta_{c}^{d}$, $R_{\hat{a} \hat{b} c d}=-2 c \delta_{c d}^{a b}$, плюс соотношения, полученные с учетом свойств симметрии и вещественности тензора $R$. Остальные компоненты этого тензора равны нулю. Следовательно, $R(X, Y) Z=c\{\langle X, Z\rangle Y-\langle Y, Z\rangle X\}, X, Y, Z \in \mathrm{X}(M)$, т.е. $M$ - многообразие постоянной кривизны $c$. Теми же рассуждениями, что и в [11], получаем, что $M$ локально эквивалентно пятимерной сфере $S^{5}$, снабженной канонической слабо косимплектической структурой.

Если же $M$ - коммутативно-киллингово $f$-многообразие нулевого постоянного типа, то из (38) и теоремы 4 легко следует, что

$$
\|X \bullet Y\|^{2}=\|X \diamond Y\|^{2}+\|X * Y\|^{2}=0
$$

и, значит, $X \diamond Y=X * Y=0 \quad(\langle\langle X, Y\rangle\rangle=0)$. Отсюда теми же рассуждениями, что и при выводе теоремы 9 , получаем, что $X \diamond Z=X * Z=0, X, Z \in \mathrm{X}(M)$. Из теорем 1 и 2 отсюда немедленно вытекает, что $\operatorname{Im} \theta=\operatorname{Im} \psi=\{0\}$, а значит, $\mathscr{D}_{P}=\{0\}$. В силу теоремы $4 M$ локально эквивалентно произведению келерова многообразия на риманово многообразие без дополнительной структуры.

Собирая полученные результаты и используя исчерпывающее описание приближенно келеровых многообразий постоянного типа, приведенное в [7], получаем следующий результат.

ТЕОРема 10. Всякое коммутативно-киллингово $f$-многообразие имеет постоянный тип тогда и только тогда, когда оно либо локально әквивалентно произведению келерова многообразия на риманово многообразие без дополнительной структуры, либо является шестимерным собственным приближенно келеровым многообразием, либо локально эквивалентно пятимерной сфере, снабженной канонической слабо косимплектической структурой. 


\section{Список литературы}

1. Gray A. Nearly Kähler manifolds // J. Different. Geom. 1970. V. 4. № 3. P. 283-309.

2. Naveira A., Hervella L. M. Shur's theorem for nearly Kähler manifolds // Proc. Amer. Math. Soc. 1975. V. 49. № 2. P. 421-425.

3. Vanhecke L., Bouten F. Constant type for almost Hermitian manifolds // Bull. Math. Soc. sci. math. RSR. 1976, 1977. V. 20. № 3, 4. P. 415-422.

4. Грицанс А. С. О геометрии киллинговых $f$-многообразий // УМН. 1990. Т. 45. № 4 . C. $149-150$.

5. Blair D. E., Showers D. K. Almost contact manifolds with Killing structure tensors. II // J. Different. Geom. 1974. V. 9. № 4. P. 577-582.

6. Кириченко B. $\Phi . K$-алгебры и $K$-пространства постоянного типа с индефинитной метрикой // Матем. заметки. 1981. Т. 29. №2. С. 265-278.

7. Кириченко В. Ф. К-пространства постоянного типа // Сиб. матем. журн. 1976. Т. 17. № 2. C. $282-289$.

8. Kiritchenko V.F. Sur le geometrie des varietes approximativement cosymplectiques // C.r. Acad. Sci., Paris. 1982. V. 295. № 1. P. 673-676.

9. Кириченко В. Ф. Квазиоднородные многообразия и обобшенные почти эрмитовы структуры // Изв. АН СССР. Сер. матем. 1983. Т. 47. №6. С. 1208-1223.

10. Kirichenko V.F. Generalized quasi-Kaehlerian manifolds and axioms of $C R$-submanifolds in generalized Hermitian geometry. I // Geometriae Dedicata. 1994. V. 51. P. 75-104.

11. Kirichenko V.F. Generalized quasi-Kaehlerian manifolds and axioms of $C R$-submanifolds in generalized Hermitian geometry. II // Geometriae Dedicata. 1994. V. 52. P. 53-85.

12. Кириченко B. $\Phi$. Аксиома $\Phi$-голоморфных плоскостей в контактной метрической геометрии // Изв. АН СССР. Сер.матем. 1984. Т. 48. № 4. С. 711-734.

13. Кобаяши Ш., Номидзу К. Основы дифференциальной геометрии. М.: Наука, 1981.

Поступило в редакцию

5.V.1998 